
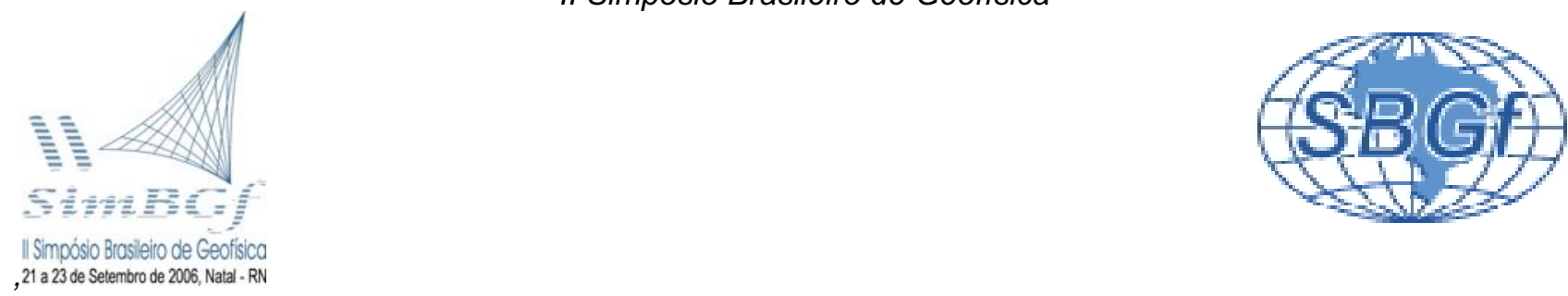

\title{
Sismicidade e Mecanismo Focal em Belo Jardim-PE
}

Afonso E. V. Lopes ${ }^{1}$, Marcelo Assumpção ${ }^{1}$, Aderson F. Nascimento ${ }^{2}$, Joaquim M. Ferreira ${ }^{2}$, José R. Barbosa ${ }^{1}$, Eduardo A.S. Menezes ${ }^{2}$, Carlos A. Anjos ${ }^{2}$, 1. IAG-USP 2. UFRN

Copyright 2006, SBGf-Sociedade Brasileira de Geofísica

Este texto foi preparado para a apresentação no II Simpósio de Geofísica da

Sociedade Brasileira de Geofísica, Natal, 21-23 de setembro de 2006. Seu conteúdo foi revisado pela Comissão Tecno-científica do II SR-SBGf mas não necessariament representa a opinião da SBGf ou de seus associados. E proibida a reprodução total ou parcial deste material para propósitos comerciais sem prévia autorização da SBGf

\section{Resumo}

Em Novembro de 2004 foi instalada uma rede de estações em Belo Jardim-PE para estudar um surto de sismicidade que persistia por vários meses. A análise dos registros indicou que o melhor modelo de velocidades tem uma camada superficial de $3 \mathrm{~km}$ com $V p=5,6 \mathrm{~km} / \mathrm{s}$ e um semi-espaço com $V p=6,1 \mathrm{~km} / \mathrm{s}$. A relação $\mathrm{Vp} / \mathrm{Vs}$ foi estimada em 1,66. O mecanismo focal corresponde a uma falha transcorrente com direção NE-SW e componente normal, possivelmente associada a uma dupla zona de fraqueza. O falhamento responsável pelos sismos observados possui potencial de gerar sismos de magnitude até $\sim 5$. A direção dos esforços na região estimada com o mecanismo focal, indica $\mathrm{SH}_{\max }$ com direção E-W.

\section{Introdução}

Em novembro de 2004 o IAG-USP e a UFRN instalaram em parceria 14 estações sismográficas em Belo Jardim (PE) para estudar a sismicidade da região. A sismicidade identificada pela UFRN em 2004, persistiu por vários meses.

A área de estudo está inserida na Província Borborema, que é conhecida por ser a região de maior atividade sísmica do Brasil. Há registros da sismicidade de Belo Jardim no Boletim Sísmico Brasileiro, que datam de 1984.

A Província Borborema (PB) é limitada a leste pelo oceano atlântico, oeste pela bacia do Parnaíba e a sul pelo Cráton do São Francisco. Trata-se de uma região com formações supracrustais extensamente deformadas por tectonismo e magmatismo policíclico (Jardim de Sá et al. 1988), podendo ser subdividida em três grandes domínios (Santos et al. 1997, Brito Neves et al. 2000): setentrional, zona transversal e meridional.

O segmento central da PB está balizado por dois expressivos lineamentos (Patos ao norte e Pernambuco, ao sul), que foram zonas de cisalhamento subparalelas E-W, dando forma retangular a esta zona. Estes lineamentos estão afastados entre si de $150 \mathrm{~km}$ em média, são observados desde a faixa costeira até a Província Parnaíba, com diversas feições estruturais e morfológicas associadas. Segundo Trompette (1994), esse conjunto de cinturões de cisalhamento são transcontinentais com continuação na África (PatosGaroua, Pernambuco-Adamauoa).

No interior da Zona Transversal destaca-se uma zona elevada, onde se podem observar interlineamentos com formas sigmoidais, com suas terminações acopladas aos lineamentos Patos e Pernambuco. A topografia e alguns lineamentos da porção norte de Pernambuco são apresentados na Figura 1.

Neste trabalho é apresentado um modelo de velocidade de ondas sísmicas determinado com dados de uma explosão de pedreira e análise de um conjunto de 20 hipocentros. Esse modelo refinado foi utilizado na determinação de hipocentros de grande precisão e na determinação do mecanismo focal. O mecanismo focal é consistente com feições topográficas e geologia.
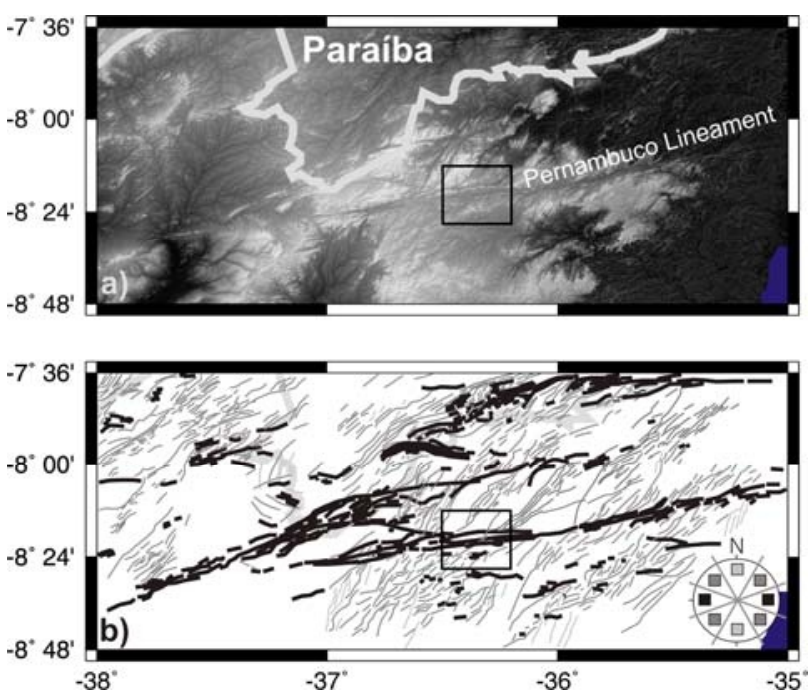

Figura 1 - Localização da área de estudo (retângulo). a) topografia da região (SRTM) $e \quad b)$ lineamentos topográficos: principais lineamentos E-W são destacados por linhas pretas grossas e lineamentos secundários por tons mais claros (veja detalhe do diagrama).

\section{Modelo de Velocidade}

Para garantir boa precisão nos hipocentros, fez-se um breve estudo para se determinar o modelo de velocidade da região. A velocidade da onda direta foi estimada com dados de uma explosão de pedreira registrada por três estações sismográficas (BJ01, BJ02 e BJ03 - Figuras 2 e 4). A posição da explosão é conhecida com boa precisão, mas a hora de origem não foi controlada. 
Como as distâncias epicentrais e chegadas da $\mathrm{P}$ nas três estações são conhecidas, pode-se calcular a hora de origem para diferentes valores de velocidade. Os valores de hora de origem calculados para cada estação são iguais para velocidade da $P$ direta igual a $5,60 \mathrm{~km} / \mathrm{s}$.

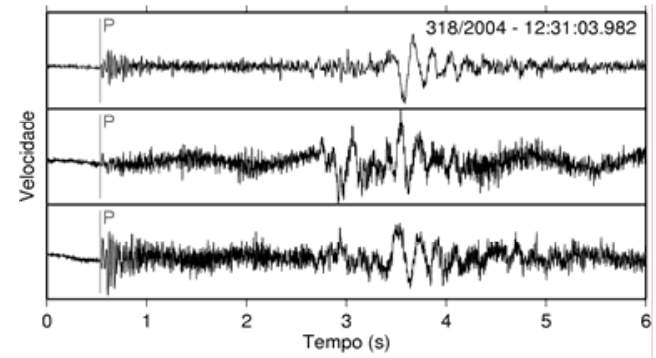

Figura 2 - Registro da explosão (BJ01) com marca da $P$. $A$ boa qualidade das ondas de superfície indica que $O$ evento realmente é superfícial. Apenas esta estação digital registrou a explosão.

A estação mais distante da explosão (26,2 km) não registrou a $P$ refratada, impossibilitando a determinação da espessura da primeira camada (só registraria se a espessura fosse inferior a 2,7 km). Análise das determinações hipocentrais feitas com algumas espessuras de primeira camada apontou uma espessura ótima de $3,0 \mathrm{~km}$. Os mesmos testes apontam uma velocidade de $6,1 \mathrm{~km} / \mathrm{s}$ para a onda $P$ no semi-espaço.

A razão de velocidades $\mathrm{Vp} / \mathrm{Vs}$ determinada com 0 diagrama de Wadati obtido com 64 dados de 20 sismos (Figura 3), foi de 1,66, inferior ao padrão (sólido de Poisson), sendo um indicativo de região com predominância de rochas félsicas.

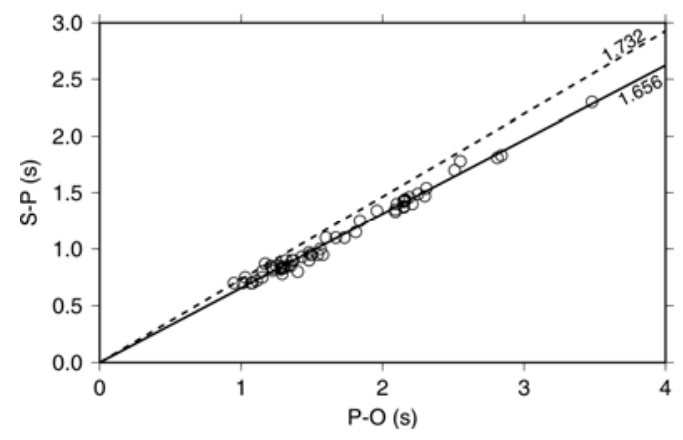

Figura 3 - O diagrama de Wadati (64 dados - 20 sismos) aponta para $\mathrm{Vp} / \mathrm{Vs}=1,656 \pm 0,014$, representado pela linha escura $(\sigma=0,05 \mathrm{~s})$. A linha tracejada é um padrão $(V p / V s=1,732)$ usado para comparar o resultado.

\section{Sismicidade}

Nos 12 dias de trabalho de campo, foram registrados mais de 600 sismos, distribuídos em dois conjuntos distintos, conforme pode ser observado na Figura 4a. Neste trabalho estudamos apenas os sismos próximos à estação BJ10. No segundo conjunto de sismos observouse apenas um hipocentro com boa qualidade, próximo a estação BJ14. As profundidade focais dos sismos estudados variam entre 3 e $6 \mathrm{~km}$, e suas magnitudes são inferiores a 3,0 na escala Richter.
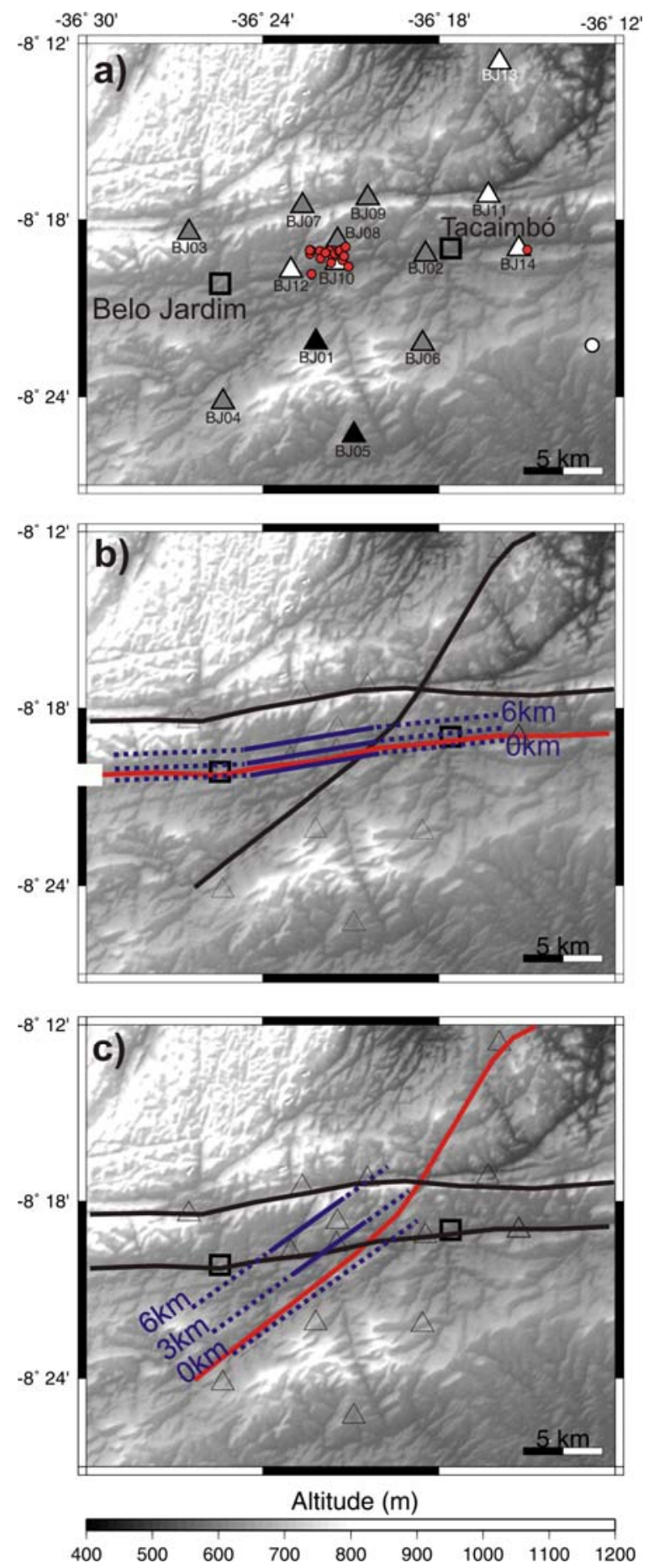

Figura 4 - Topografia da área estudada (tons de cinzas). a) Triângulos pretos são estações digitais com relógio aferido, brancos são sem GPS e cinzas são estações analógicas relógio aferido. Círculos vermelhos representam epicentros e branco a explosão. Quadrados são cidades. Em b) e c) os principais lineamentos são destacados por linha preta e lineamento que explica o resultado do mecanismo focal é dado pela linha vermelha. Linhas azuis continuas são profundidades do plano de falha das soluções do b) Grupo1 e c) Grupo2, e as linhas tracejadas a extrapolação deste plano. 


\section{Mecanismo Focal}

Registraram-se mais de 600 sismos no período de estudo, mas apenas 70 por mais de 3 estações, e destes, apenas 10 possuem mais de 10 leituras e resíduo $(\mathrm{rms})$ inferior a 0,05 segundos. As polaridades das ondas $P$ e $\mathrm{SH}$, e razão de amplitudes (SH/P) desses 10 sismos foram utilizadas no estudo do mecanismo focal com o programa FocMec (Snoke et al. 1984). As soluções permitem um erro de polaridade de $\mathrm{P}$ e nenhum de $\mathrm{SH}$. O limite máximo para resíduos da razão de amplitude $\log (\mathrm{SH} / \mathrm{P})$ foi de 0,4 .
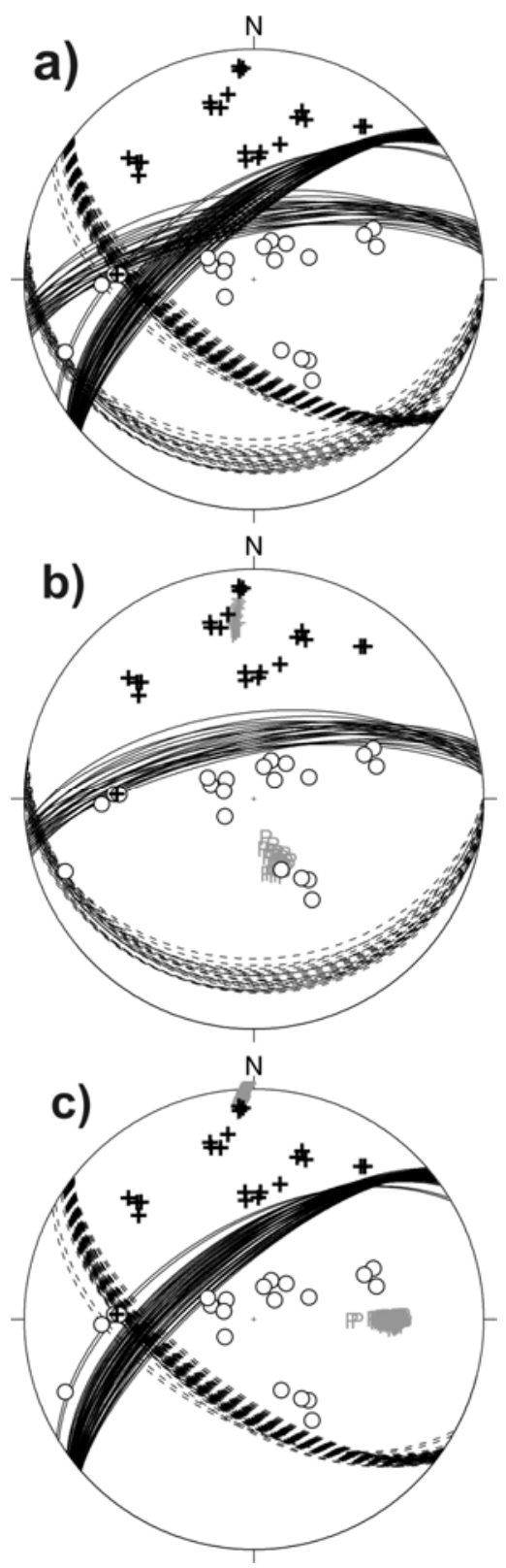

Figura 5 - a) Todas as soluções obtidas com polaridades de ondas $P$, SH e razão de amplitude (SH/P), mostrando dois grupos de soluções similares. b) Grupo (1) de falhas normais E-W. c) Grupo (2) de falhas transcorrentes NESW com componente normal. Letras representam eixos de pressão $(P)$ e tração $(T)$.
Os resultados obtidos com o FocMec são divididos em dois conjuntos principais (Figura 5a), os quais indicam falhamento normal E-W (Figura 5b) e falhamento transcorrente NE-SW com componente normal (Figura 5c).

A principio o resultado mais plausível, visto a proximidade com o lineamento Pernambuco, seria o grupo de falhas E-W. Por outro lado, a geologia indica que o lineamento Pernambuco foi composto por falhas transcorrentes, o que é evidenciado pelas estruturas sigmoidais entre ele o lineamento Patos.

Como se pode observar na Figura 6, a distribuição dos hipocentros é compatível com ambas às soluções, porém a segunda solução (falha NE-SW) é mais compatível com a solução independente obtida apenas com hipocentros. A extrapolação de ambas as soluções para um plano de falha que corta a superfície é consistente com lineamentos topográfico observado na superfície (Figura 4). Portanto, a primeira solução é compatível com todos os dados e poderia ajudar a explicar a ocorrência de sismos próximos à estação BJ14, e a segunda solução é compatível com os antigos movimentos que de transcorrencia destral dos lineamentos Patos e Pernambuco.

A média do primeiro conjunto de primeira solução indica uma falha normal com azimute $260,0^{\circ}$, mergulo $73,0^{\circ}$ e rake de $-94,20^{\circ}$. O segundo conjunto indica uma falha transcorrente com azimute $232,3^{\circ}$, mergulho de $61,8^{\circ}$ e rake de $-147,5^{\circ}$
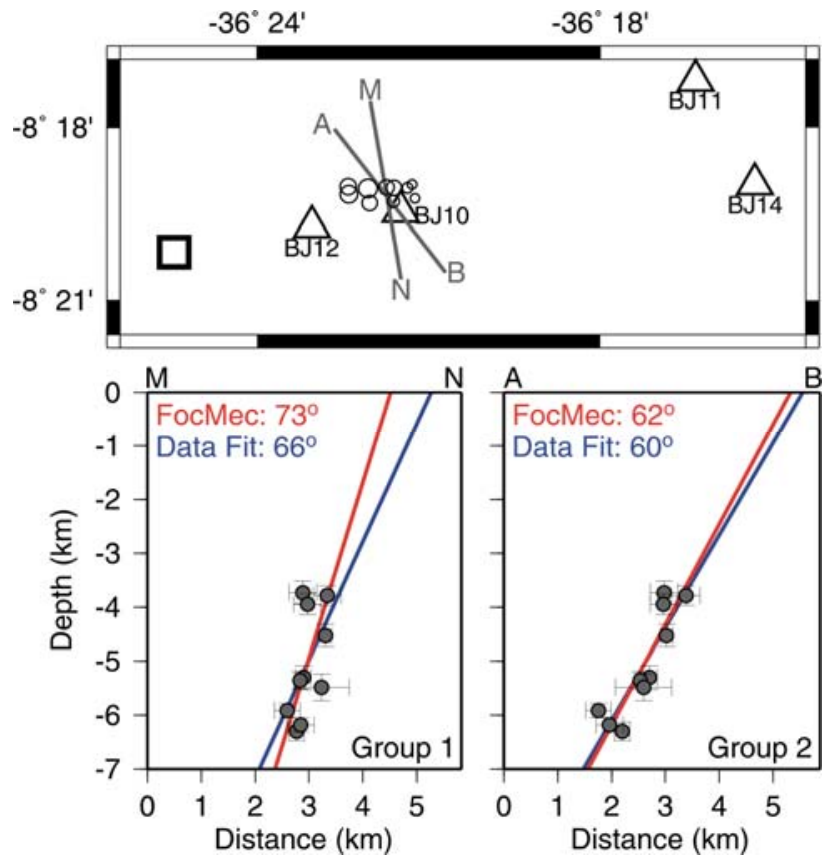

Figura 6 - Círculos são hipocentros dos sismos com mais de 10 leituras (tamanho proporcional a profuncidade), os triângulos as estações e o quadrado a cidade de Belo Jardim. Os perfis $\mathrm{M}-\mathrm{N}$ e $\mathrm{A}-\mathrm{B}$ são perpendiculares às soluções das Figuras $5 b$ e $5 c$, respectivamente. Os perfis são apresentados junto ao ajuste do plano de falha com os hipocentros (Data Fit) e com o mecanismo focal. 
Ambas as soluções são bastante precisas, com erros da ordem de $2^{\circ}$ no azimute, $4^{\circ}$ no mergulho, e $5^{\circ}$ no rake. $\mathrm{Na}$ primeira solução (Figura $5 b$ ), o eixo de pressão $(P)$ é quase vertical, e o de tração (T) é sub-horizontal com direção N-S. Na segunda solução (Figura $5 c$ ), o eixo $P$ é sub-horizontal com direção $\mathrm{E}-\mathrm{W}$, e o eixo $\mathrm{T}$ é horizontal com direção N-S. A estimativa da direção dos esforços com o eixo $B$ da falha normal e $P$ da transcorrente, indica nos dois casos, compressão $\sim$ E-W.

\section{Conclusões}

Como se pode observar nas Figuras 4 e 6, é difícil evidenciar qual é o mecanismo focal mais adequado para a região. Dados geológicos mostram que os movimentos antigos são compatíveis com o segundo caso, mas os epicentros próximos a estação BJ14 podem ter sido gerados pela mesma falha E-W evidenciada na Figura 4. Ambos os mecanismos focais estudados satisfazem bem os dados observados, embora o segundo conjunto de soluções seja mais compatível com a solução independente obtida apenas com a distribuição dos focos.

O mecanismo focal de falha transcorrente NE-SW com componente normal pode ser explicado pelos movimentos transcorrentes gerados por zonas de franquezas sigmoidais criadas pelos antigos movimentos dos lineamentos Patos e Pernambuco. Esse resultado nos permite abordar algumas idéias maiores e interessantes relacionadas à estrutura de lineamentos da região. Se admitirmos que a falha estudada faz parte de uma estrutura sigmoidal (comum na região) causada por falhamentos maiores, podemos admitir que os sismos têm origem em movimentos antigos de falhas maiores, ou eventual reativação de falhas antigas. Esse modelo seria compatível com o tipo de estruturas observadas na região (Figura 7). O mais provável é que o estado de esforços neotectônicos (compressão E-W na região) tenha atuado principalmente nessas fraquezas interlineamentos, já que os grandes falhamentos são paralelos ao $\mathrm{SH}_{\max }$.

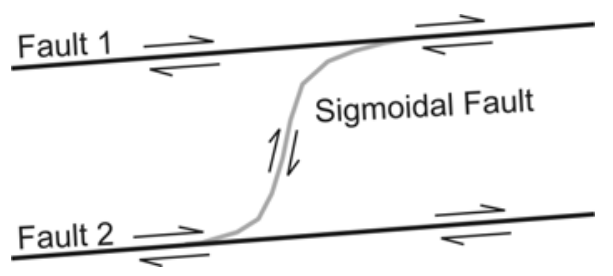

Figura 7 - Estrutura sigmoidal sendo ativada por movimentos de falhas mestras.

Neste ponto é válido salientar que todo o nordeste do Brasil apresenta pequenas estruturas NE-SW, em geral ofuscadas pelos mega lineamentos E-W. Possivelmente esses falhamentos menores devem ser vistos com mais atenção que as mega-estruturas, que no atual estado de esforços podem não estar otimamente orientadas para gerar sismos.

O mecanismo focal de falha normal E-W também é bastante consistente e pode estar evidenciando a movimentação da zona de fraqueza que dá origem ao lineamento Pernambuco. Neste caso, a movimentação pode estar ocorrendo devido a um processo de acomodação de deformações antigas ou devido a ação de tração N-S. Como o teto da falha está descendo e a região adjacente ao muro é mais baixa, podemos concluir que a porção mais alta do lineamento esta descendo. Ou seja, a porção adjacente ao teto foi anteriormente elevada pelas tensões tectônicas, e atualmente pode estar descendo devido a ausência de grandes esforços compressivos N-S. Portanto, esse processo pode ser tratado como uma reativação neotectônica sob esforços atuais diferentes.

Ao levantarmos a questão de risco sísmico em Belo Jardim, é válido salientar que não importa realmente qual solução representa a falha que provocou os eventos, pois devemos considerar que ambas são evidentes na superfície e que a ocorrência dos sismos possivelmente estar ligada a uma dupla zona de fraqueza. Deste modo, ambas as estruturas são responsáveis pela ocorrência dos eventos, já que o movimento da falha ativa pode ter sido estimulado pela conjunção das duas zonas de fraqueza.

Os movimentos de acomodação de uma falha E-W não são realmente preocupantes. Por outro lado, a falha transcorrente proposta na segunda solução, teria área da ordem de $300 \mathrm{~km}^{2}$, e potencial de gerar sismos de magnitudes próximas de 5,0 na escala Richter.

\section{Agradecimentos}

A Marcelo Bianchi pela instalação e configuração do notebook utilizado no campo, e pelo apoio e assistência com softwares. A Newton Campos Jr. pela assistência e discussões sobre algoritmos em C\#. Esse projeto contou com apoio da FAPESP (03/12204-8) e ADIMB (através do Fundo CT-Mineral).

\section{Referências}

Brito Neves, B.B., Santos, E.J., Van Schmus, W.R., 2000. Tectonic history of the Borborema Province, In: Cordani, U.G., Milani, E.J., Thomaz Filho, A., Campos, D.A. (Ed.), Tectonic Evolution of South America, $31^{\text {st }}$ International Geological Congress.

Jardim de Sá, E.F., Legrand, J.M., McReath, E.I., 1988. Estratigrafia de rochas granitóides na região de Seridá (RN-PB) com base em critérios estruturais, Revista Brasileira de Geociências, v. 18(1): 50-57.

Santos, E.J. \& Medeiros, V.C., 1997. Constraints from granitic plutonism on Proterozoic crustal growth of the Zona Transversal domain, Borborema Province, NE, Brazil", II ISGAM, Salvador(BA), Brasil, 237-239.

Snoke, J.A., Munsey, J.M., Teague, A.C., Bollinger, G.A., 1984. A program for focal mechanism determination by combined use of polarity and SV-P amplitude ratio data, Earthquake note, 55.

Trompette, R., 1994. Geology of Western Gondwana, Pan-African - Brasiliano aggregation of South America and Africa", A.A. Balkema, Rottrerdam, Brookfield, 350p. 